Cinémas

Revue d'études cinématographiques

Journal of Film Studies

\title{
La voix off dans Sunset Boulevard
}

\section{Paul Warren}

Volume 5, numéro 3, printemps 1995

\section{Cinélekta 1}

URI : https://id.erudit.org/iderudit/1001147ar

DOI : https://doi.org/10.7202/1001147ar

Aller au sommaire du numéro

Éditeur(s)

Cinémas

ISSN

1181-6945 (imprimé)

1705-6500 (numérique)

Découvrir la revue

Citer cet article

Warren, P. (1995). La voix off dans Sunset Boulevard. Cinémas, 5(3), 65-75. https://doi.org/10.7202/1001147ar

\section{Résumé de l'article}

La voix off, errant en bordure du film, possède d'autant plus de pouvoir énonciatif qu'elle refuse de s'incarner dans le donné factuel audiovisuel. Le cinéma classique hollywoodien a développé une série de procédures pour limiter ce pouvoir, cette possibilité qu'a la voix off, libérée de l'image d'un corps, de bloquer ou de fractionner le déroulement du récit. L'analyse de Sunset Boulevard (Wilder, 1950) permet de débusquer ces procédures visant essentiellement à intégrer la voix dans le corps de la diégèse, à faire " coulisser » la voix off dans la voix in. 


\section{La voix offdans Sunset Boulevard}

\section{Paul Warren}

\section{RÉSUME}

La voix off, errant en bordure du film, possède d'autant plus de pouvoir énonciatif qu'elle refuse de s'incarner dans le donné factuel audiovisuel. Le cinéma classique hollywoodien a développé une série de procédures pour limiter ce pouvoir, cette possibilité qu'a la voix off, libérée de l'image d'un corps, de bloquer ou de fractionner le déroulement du récit. L'analyse de Sunset Boulevard (Wilder, 1950) permet de débusquer ces procédures visant essentiellement à intégrer la voix dans le corps de la diégèse, à faire "coulisser " la voix off dans la voix in.

\section{ABSTRACT}

Voice over, a voice that hovers in the margins of a film, possesses all the more enunciatory power for its refusal to be embodied in gross audiovisual data. Classic Hollywood cinema developed a series of procedures to limit this power, this potentiality for voice over, freed from the image of a body, to block or fragment the unfolding of the plot. An analysis of Sunset Boulevard (Wilder, 1950) enables the author to unmask these procedures, whose aim is essentially to integrate the voice over into the body of the diegesis, to squeeze the voice over into the frame.

La voix off dans le cinéma de fiction hollywoodien est rarement une voix sans tête et sans corps, une voice over surplombant l'histoire, l'observant et la "pro-férant" de haut et de loin sans jamais y descendre (comme c'est le cas pour la voix off de certains films de Fellini ou de Godard). Elle a peu affaire avec le discours off du film documentaire, du film documentaire poli- 
tique; cette "voix de Dieu" comme on l'appelle a d'autant plus de pouvoir énonciatif qu'elle refuse de s'incarner dans le donné factuel audiovisuel, se donnant de la sorte (et parfois superbement) toute liberté de l'orienter, ce donné, selon son bon vouloir idéologique. Et nous savons avec Pascal Bonitzer qu'elle peut se payer ce luxe, pour la bonne raison que, sans elle, le donné factuel du documentaire est, bien souvent, de l'ordre de l'indécidable'.

La voix off du fictionnel classique hollywoodien est une voix intégrée ou plutôt, c'est une voix qui cherche constamment à s'intégrer. Mieux, c'est une voix qui fait tout pour se faire in, comme pour se faire pardonner ses fugues dans le off. C'est habituellement la voix du personnage principal dont les techniciens cherchent à faire oublier qu'elle est asynchrone. Et c'est de bonne guerre pour un cinéma dont la règle d'or et la marque de fabrique ont toujours été de dissimuler ses codes et d'effacer les traces de sa production manufacturée, en vue d'assurer un meilleur synchronisme entre l'écran lumineux et la salle obscure.

Je me propose ici d'étudier le système d'intégration de la voix off, la façon dont s'y prend la voix off pour se faire in (dans les deux sens du terme), et cela à travers le film de Billy Wilder, Sunset Boulevard (1950). Pourquoi Sunset Boulevard? D'abord, parce que c'est un film important et bien connu des cinéphiles. Ensuite, parce que c'est un film où le réalisateur s'est donné les meilleures chances du monde de sortir la voix off des conventions narratives des studios d'Hollywood. En effet, Wilder, avec ce film, cherchait à renouveler la production hollywoodienne en crise. Nous sommes au début de la décennie cinquante: l'euphorie pour le nouveau medium télévision, une certaine influence du film européen aux États-Unis ainsi que la loi antitrust qui venait de contraindre les Majors à se séparer de leurs salles obligeaient les cinéastes américains à se dépasser et, dans le meilleur des cas, à remettre en question certaines conventions des grands studios. En tout cas, Wilder a utilisé la voix off dans Sunset Boulevard avec une audace qui ne lui est pas coutumière. Il fait "dé-tonner" la voix off pour étonner le spectateur, pour le réveiller de sa torpeur. Il s'agit d'une voix d'outre-tombe. C'est la voix d'un mort qui, de manière assez invraisemblable, accom- 
pagne les trois quarts des séquences du film. Wilder voulait tellement faire original qu'il s'est risqué à tourner la première séquence de son histoire dans une morgue, où le cadavre du héros se dressait sur sa couche et se mettait, en voix off, à raconter sa pauvre vie aux autres cadavres tout autour. Devant les rires ironiques des spectateurs de la projection pilote qu'il avait organisée pour tester son film, Wilder a remplacé la séquence par celle qui ouvre désormais le film et dont toute la critique a souligné la grande originalité: la voix off du héros qui nous raconte, à la première personne, comment sa lamentable aventure a commencé, cependant que son propre cadavre flotte dans la piscine d'un riche manoir de Beverly Hills.

Voici, pour la bonne compréhension de mon analyse, un bref résumé de cette lamentable aventure narrée en voix off par un mort. Joe Gillis (William Holden), le défunt qui raconte son aventure, est un scénariste en mal d'écriture et d'emploi ad hoc. Il échoue par hasard dans la propriété princière de la grande star du muet, Norma Desmond (Gloria Swanson). Après quelque résistance de forme, Gillis accepte, par besoin d'argent et de

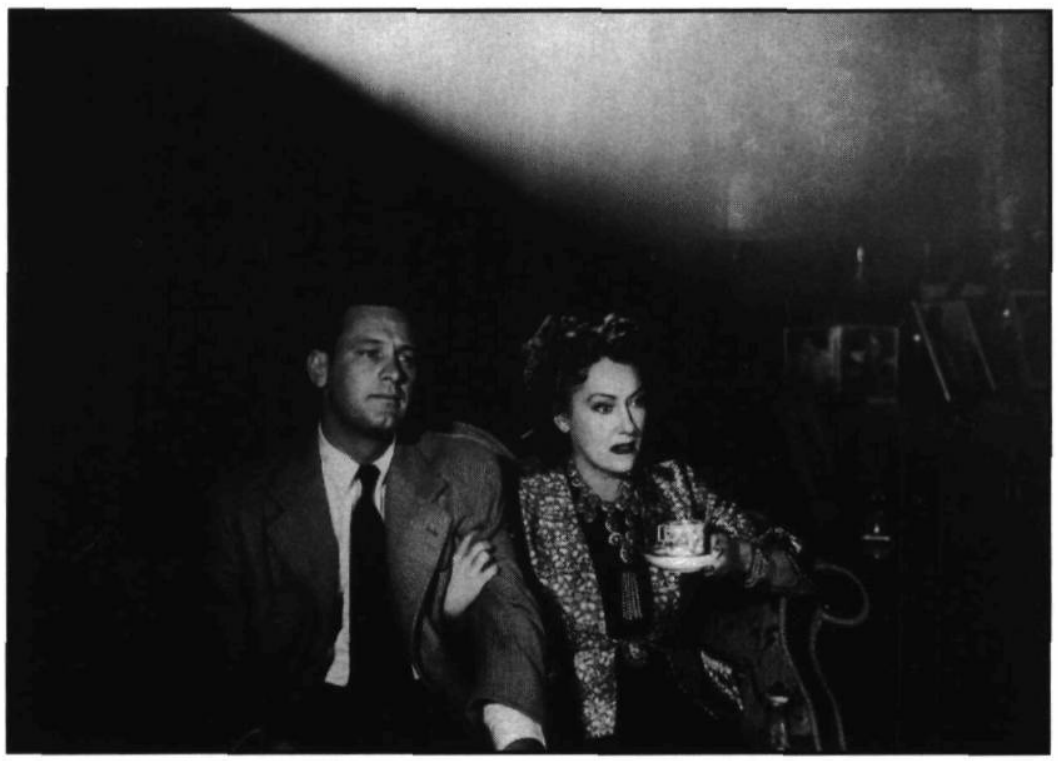

Sunsef Boulevard de Billy Wilder (1950)

Collection Cinémathèque québécoise 
confort, de vivre au manoir de Norma Desmond le temps de réécrire le scénario sans valeur que celle-ci a conçu dans l'obsession de son retour en force à l'écran. Joe devient le gigolo de la star vieillissante, laquelle se révèle jalouse et suicidaire. De plus en plus dégoûté de lui-même et ayant rencontré une jeune scénariste avec laquelle il travaille, la nuit, en secret, à un scénario qui lui tient à cœur, Joe décide de quitter Norma. Celle-ci, devenue folle, le tue.

Ce que je veux montrer ici, c'est comment la voix off, qui pourtant a voulu se placer à une distance de l'autre monde de son objet, s'est prise pour faire oublier son éloignement, pour se prendre à l'histoire linéaire, ponctuelle et terre à terre, pour réintégrer le corps de la diégèse en retrouvant le corps du personnage. En d'autres termes, ce que je veux démontrer en analysant la voix off de ce film particulier, Sunset Boulevard (que les Français, soit dit en passant, ont malencontreusement traduit par Le Boulevard du crépuscule), c'est que même dans le cas où Hollywood met toutes les chances de son côté pour jouer de la distanciation, il prouve encore son anti-brechtisme fondamental. Disons mieux: Hollywood récupère, concrétise et américanise tout, même the voice of God.

La voix off, nous l'avons mentionné, se fait entendre dès les premières images du film. C'est la voix commentatrice de William Holden sur le cadavre de son personnage, Joe Gillis, qui flotte dans la piscine de Norma Desmond. Une voix connue du public (Holden est une star hollywoodienne depuis dix ans déjà, depuis le film Golden Boy, 1939, de Mamoulian). Cette voix nous entraîne dans un flash-back qui va durer tout le film, sans discontinuité visuelle. "Comment ai-je échoué dans cette piscine fashionable?" nous dit, grosso modo, la voix. "Il faut, pour comprendre, continue-t-elle, revenir six mois en arrière..." C'est le flash-back. Et là, nous voyons William Holden jouant en voix synchrone le rôle d'un scénariste, Joe Gillis, qui se démène pour trouver un job payant et régler ses dettes.

Ce qu'il importe de bien saisir pour suivre l'analyse, c'est ceci : dès le départ de l'histoire de Gillis en flash-back s'installe (et cela jusqu'à la dernière séquence du film) un constant va-etvient entre le off et le in, entre la voix asynchrone et la voix syn- 


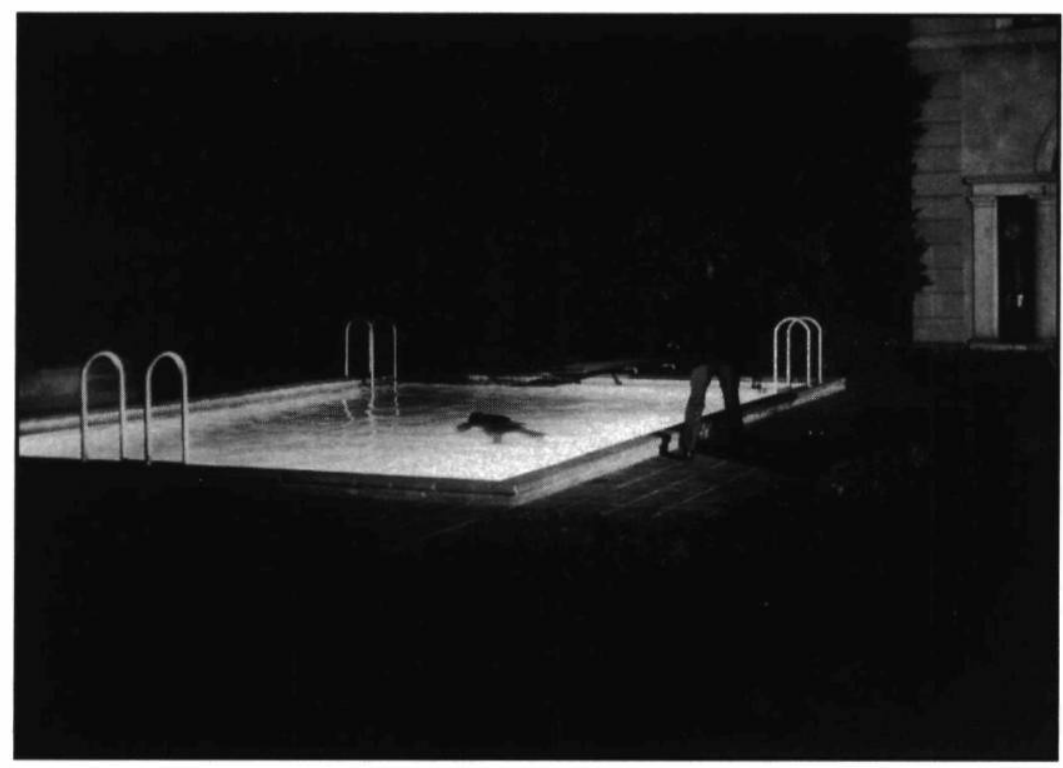

\section{Sunsef Boulevard de Billy Wilder (1950)}

Collection Cinémathèque québécoise

chrone de Gillis, entre la voix au passé du défunt Gillis et celle du Gillis bien présent et bien vivant. À telle enseigne que cette structure vocale binaire qui fonctionne au rythme et sur le mode du champ-contrechamp intégrateur, en point-contrepoint sonore, pour ainsi dire, en arrive, comme c'est le cas de toutes les techniques cinématographiques hollywoodiennes, à se rendre pratiquement invisible en entrainnant progressivement le off à coulisser à notre insu dans le in.

Mais entrons dans le film, un peu à la manière du off qui pénètre dans le $i$. La voix off fait office de monologue intérieur. C'est la voix de Joe Gillis qui réfléchit sur son propre comportement et sur celui des autres protagonistes du drame. Elle implique, par conséquent, la présence de Joe dans le champ ou, parfois, dans un hors-champ qui voisine immédiatement le champ d'un personnage auquel il réagit (sur lequel il fait porter sa réflexion).

Lorsque la voix off se fait entendre sur/dans un plan d'ensemble ou de demi-ensemble (ce qui se produit beaucoup dans 
le film), il s'agit, très souvent, d'un plan de transition, de déambulation, de passage d'un espace à un autre. Cela est de règle, à ma connaissance, dans le cinéma américain de fiction utilisant la voix off. Celle-ci joue ainsi le rôle de la musique qui, conventionnellement et... imperceptiblement, remplit le vide ou le relâchement de la diégèse. D'ailleurs, l'on constate à l'étude que la voix off sur plan d'ensemble de voyagement dans Sunset Boulevard s'accompagne, presque toujours, d'une musique en background, musique qui, habituellement, précède et suit de quelques secondes sa présence dans le plan, comme pour faire baigner la voix dans un cocon sonore. La musique fait se lever la voix off qui accompagne la déambulation de Gillis (par exemple, vers le lieu où l'attend sa jeune collègue scénariste) et vient déposer cette même voix off dans la séquence suivante (en l'occurrence, dans le studio de scénarisation), où la voix in (après quelques secondes de musique seule dans le plan) prend la relève. Ajoutons que l'utilisation de la technique de l'enchaîné permet, tout au long du film (et singulièrement dans les séquences de transition), de fondre en douceur la voix off, déjà en synchronisme avec la musique, dans le in de l'histoire au présent.

Il faut noter que la musique extradiégétique qui accompagne la voix off s'avère, à quelques reprises, être une musique intradiégétique: notamment, quand Gillis se rend au salon pour rejoindre Norma Desmond, on entend, en background de son monologue sur l'étrangeté de sa situation, une musique d'orgue; or, cette musique se fait de plus en plus forte au fur et à mesure de la progression de Gillis, jusqu'au moment où nous découvrons que c'est Max (Eric von Stroheim), le serviteur de la star, qui est le musicien. La musique off est aspirée par la musique in, à la manière de la voix off monologuée qui se mue en dialogue in. Un point est à préciser concernant la déambulation en plan d'ensemble dans Sunset Boulevard. Dans certaines séquences, tout semble propice à l'intervention de la voix off, singulièrement dans la séquence où Norma Desmond, qui joue au bridge avec des invités, demande à Gillis d'aller vider le cendrier; le scénariste, ravalé au rang de serviteur, s'exécute avec un regard qui en dit long sur sa frustration; dans un plan éloigné, il traverse le salon accompagné de la musique ambiante qui prend 
une note dramatique. Cependant, la musique n'a pas fait lever, cette fois, la voix off. Elle est seule à commenter la scène, ce qui est habile de la part de Wilder: le spectateur a le loisir de projeter sur la situation son propre monologue intérieur. Cela laisse entendre que la voix off, dans un film hollywoodien de fiction, est un élément bien peu structurant de l'articulation narrative. Elle n'a pas grand-chose à voir avec la voix off du documentaire qui, de fait, est une aide importante que le film se donne de l'extérieur pour lui permettre de tenir ensemble des données factuelles qui risqueraient, sans elle, de sombrer dans l'incohérence ou l'indécidable.

Mais c'est lorsque la voix off est proférée en plan rapproché ou en gros plan que le travail de "coulissage" de Wilder et de son équipe devient intéressant. Il se passe là des échanges entre le off et le in, entre le champ du off et le champ du in, d'une tenue professionnelle à laquelle seuls des techniciens qui peaufinaient depuis un demi-siècle déjà la stratégie du champcontrechamp et, singulièrement, du shot-reaction shot pouvaient accéder.

Voyons cela de près. Je mettrai l'accent sur les séquences où le "coulissage" entre le off et le in me paraît si bien réussi qu'il devient difficile de percevoir le passage de l'un à l'autre et vice versa. Il est évident que cet exercice auquel je me livre veut aller au-delà de l'analyse formelle afin de mettre en valeur le plaisir de l'analyse formelle. En expliquant comment la voix off se prend dans la voix in, techniquement, je veux montrer d'une part la fascination de l'écranique américain pour le spectateur de la salle obscure et, d'autre part, le piège dans lequel va inexorablement se prendre le héros de Sunset Boulevard.

Dans la séquence où Gillis, au volant de sa vieille décapotable, s'engage dans Sunset Boulevard (le nom du célèbre boulevard de Los Angeles est clairement inscrit sur un panneau indicateur), sa voix off se fait entendre qui vient rejoindre la musique déjà présente en background depuis le début de la séquence. Remarquons que nous avons entendu la voix off auparavant, à plusieurs reprises même, depuis les dix minutes que le film est en marche. Mais c'était une voix off en surplomb, du type de l'accompagnement musical ou documentariste, une voix 
off qui relayait les voix in dialoguées, planant sur l'histoire qui s'amorçait au ras du sol. Bref, une voix off qui se faisait "voir" en tant que off, avant d'atterrir dans le in du film. Il n'en reste pas moins que ces séquences en off- pendant les dix premières minutes que prend le film à introduire le personnage principal et son idiosyncrasie - fonctionnent en alternance avec les séquences en in, inscrivant déjà dans l'écran, mais de l'extérieur encore, l'importante scansion du off et du in que le reste du film va se charger d'intégrer et d'intérioriser structurellement.

Avec Gillis qui s'engage dans Sunset Boulevard, le film, véritablement, commence à opérer et tous les patterns doivent être parés pour jouer leur rôle respectif: le contrechamp par rapport à son champ, le reaction shot en regard de son shot et, bien sûr, le off dans sa relation à son in. Tout est prêt pour l'action: Gillis n’a pas de contrat de scénarisation, il a des arrérages de $300 \$$ sur sa bagnole, les huissiers sont à sa poursuite et il cherche désespérément de l'argent. Roulant dans Sunset Boulevard, il fait, en off, le bilan de son triste sort qui l'accule à se crétiniser dans un job de fonctionnaire à $35 \$$ par semaine. La caméra en travelling arrière nous le montre de face. La voix "musicalise" bien sagement à sa place off, comme à son habitude depuis le début du film. Mais voilà que Gillis, en regardant dans son rétroviseur, entrevoit les deux huissiers dans leur voiture, qui l'ont reconnu et qui le prennent en chasse. La voix off est stoppée, la musique fait un bond en avant, la caméra se rapproche un moment du visage de Gillis, puis fait un brusque zip pan sur la voiture des poursuivants. À ce moment précis, sur ce plan de la voiture des huissiers, on entend la voix de Gillis dire, simplement, "oh! oh!». Est-ce toujours la même voix off de Gillis qui continue, non plus sur lui-même conduisant, mais sur les huissiers dans leur voiture? Est-ce plutôt la voix de Gillis qui s'est faite in (qui s'est faite lipsync) et que l'on entend en off? Impossible de le dire. Ce que l'on est en mesure de dire, cependant, c'est que Wilder, d'entrée de jeu, nous embarque dans son histoire en jouant sur tous les plans, y compris celui de la voix off?

Analysons d'autres séquences. Max, le serviteur de Norma Desmond, installe Gillis, qui vient de se faire embaucher comme scénariste, dans une chambre au-dessus du garage. Gillis dit à 
Max, faisant référence à son entrée impromptue dans le chemin conduisant au manoir (il fuyait les huissiers) : "Je me suis engagé dans une voie intéressante." Et Max de répondre: "Très juste, monsieur, bonsoir!» Alors, Gillis, regardant le serviteur quitter la chambre, se dit par-devers lui-même: "Je me suis mis à penser qu'il était un peu cinglé lui aussi." La voix off de Gillis réagit à la voix in de Max, en parfaite continuité. Il n'y a pas eu de rupture, ni de plan, ni de ton, ni d'éclairage. On est seulement passé en douce du présent de l'indicatif au passé et la musique est intervenue, se levant dans le background pour faire mieux " coulisser" la voix off.

Mais ce qui rend singulièrement fluide la coulée de la voix off "remémorisante" dans le présent de l'histoire remémorée, c'est lorsqu'elle se fait - et ça se passe ainsi dans de nombreuses séquences du film — son contrechamp et son contrepoint, s'insinuant ainsi dans la structure la plus efficace de la narrativité cinématographique : le champ-contrechamp. Dès lors, la voix off intervient en glissant dans la séquence, pour en devenir (pour nous spectateurs, le temps d'une réplique) partie constitutive. "Veux-tu de ce boulot, oui ou non?", demande Norma Desmond à Gillis (la caméra est sur elle). "Oui, je le voulais ce boulot... et en finir au plus vite", de monologuer le scénariste (la caméra est sur lui). Là encore, la voix off réagit à la voix in, mais parce qu'elle épouse la structure conventionnelle et parfaitement intégrée du contrechamp par rapport à son champ, elle en arrive à passer pour et comme du in.

Il y a des stratégies de champ-contrechamp plus sophistiquées pour "gommer" le off, mieux, pour le travestir en in. Par exemple, la voix off de Gillis peut prendre la relève de la voix d'un personnage qui a basculé dans le hors-champ, d'une voix, par conséquent, qui est devenue elle-même off. À preuve, la séquence où Norma Desmond dit à Gillis (la caméra est sur Norma) que point n'est besoin de deux voitures et que la sienne suffit. La caméra vient cadrer une antique automobile remisée dans le garage, et l'on entend en off (sur le plan de l'auto) la voix de Norma qui dit : "Elle m’a coûté $28000 \$$ »; alors, la musique de transition se lève et l'on voit (en fondu enchaîné qui efface la coupure) la même automobile, mais cette fois, toute rutilante, 
qui roule à nouveau et sur laquelle on entend, après deux secondes de musique seule, la voix off de Gillis qui dit: "Eh bien, Max a astiqué la vieille bagnole et l'a remise sur ses roues." Deux voix qui se répondent dans le off cependant que l'on passe subrepticement d'une séquence à l'autre.

Il faut dire que ce binarisme structurel éminemment "coulissant " du champ-contrechamp sied bien à ce film qui, je dirais, "marche sur le bout des pieds pour ne pas déranger le spectateur ", pour lui rendre vivante et bien réelle et ainsi lui faire avaler une voix off qui, quoi qu'elle dise et de quelque manière qu'elle le dise, risque toujours de montrer ce qu'elle est : la voix d'un défunt. Cette voix off est chuchotée, en sourdine, comme si elle cherchait à se faire oublier. Elle est de même nature que la caméra qui se déplace avec les personnages, annulant de la sorte ses propres mouvements, des mouvements glissants, qui se faufilent à pas feutrés, dans les pièces, dans les escaliers et les corridors du vieux manoir délabré. Elle cadre bien avec l'éclairage qui rend les êtres et les objets un peu vagues, un peu flous, comme perçus dans un clair-obscur expressionniste. Elle colle à la musique de piano et d'instruments à cordes qui se tient en coulisse pour mieux se glisser dans l'image en faisant comme si elle n'y était pas. En fait, la musique musicalise la voix off en l'accompagnant la plupart du temps. Quand elle se fait plus forte, entraînant dans son sillage un travelling rapide ou un zoom in, c'est souvent pour porter sur ses notes le crescendo de la voix off, laquelle a des chances alors de passer inaperçue.

Nous avons parlé du champ-contrechamp comme structure de cohésion entre la voix off et le dialogue in. Ce qui m'amène à dire un dernier mot sur le rapport regardé/ regardant. Les séquences les plus nombreuses et les plus dramatiques de Sunset Boulevard sont, bien sûr, celles qui mettent en présence Joe Gillis et Norma Desmond. Or, ces séquences (nous en avons vu un exemple dans la scène de l'offre d'emploi) exigent une mâ̂trise particulière des patterns cinématographiques pour que le off monologué puisse s'intégrer sans heurts dans les rapports dialogués et réalistes des personnages. C'est le regard de Gillis qui est privilégié. Forcément, c'est lui qui rapporte son aventure personnelle. Son regard se pose sur Norma Desmond, sur Max 
lorsqu'il est dans son environnement immédiat et sur le mobilier du manoir tout autour. C'est le regard de Gillis qui note le comportement et les regards sur lui de Norma. C'est la trajectoire de son regard qui fait se lever la voix off (parfois quelques mots seulement perdus au milieu du in), laquelle vient parfois se déposer à l'insu de Norma toujours, à notre insu presque, entre deux phrases et deux regards in.

Me frappe dans ce film et particulièrement dans les séquences qui mettent en jeu les deux personnages principaux ce que j'appellerais l'" entre-deux" de la voix off, comme si Wilder avait voulu l'emprisonner et la couler dans le temps présent de l'histoire fictionnelle.

Étrangement, ce film, qui s'était pourtant donné une voix pour que l'on puisse observer sur l'écran le phénomène de l'aliénation du star system, fait tant et si bien qu'il en arrive à absorber le spectateur dans l'écran à un point tel qu'il ne puisse plus voir ni entendre que la petite histoire ponctuelle du scénariste Joe Gillis, qui se prend aux pièges d'une vedette vieillissante et hystérique du nom de Norma Desmond, alias Gloria Swanson.

Université Laval

\section{NOTES}

1 Voir Serge Toubiana dans "Savoir posthume: La Spirale", Cahiers du Cinéma, n'265 (1976).

2 Il m'a toujours semblé que la séquence de poursuite au début du film $A$ bout de souffle (1959) de Godard est une parodie de cette séquence qui amorce le drame de Sunset Boulevard. Tout y est, mais scandaleusement heurté et distancé : la voix du héros qui parle tout haut (en lipsync) au volant de sa voiture volée, le rétroviseur, la police, le "merde les flics", la poursuite... et la bifurcation brutale dans une voie secondaire le long de la route principale... 